\title{
Prevalence and correlates of explosive anger among pregnant and post-partum women in post-conflict Timor-Leste
}

\author{
Derrick Silove, Susan Rees, Natalino Tam, Mohammed Mohsin, Alvin Kuowei Tay and Wietse Tol
}

\section{Background}

Little is known about explosive anger as a response pattern among pregnant and post-partum women in conflict-affected societies.

\section{Aims}

To investigate the prevalence and correlates of explosive anger among this population in Timor-Leste.

\section{Method}

We assessed traumatic events, intimate partner violence, an index of adversity, explosive anger, psychological distress and post-traumatic stress disorder among 427 women (257 in the second trimester of pregnancy, 170 who were 3-6 months postpartum) residing in two districts of Timor-Leste (response $>99 \%$ ).

\section{Results}

Two-fifths (43.6\%) had explosive anger. Levels of functional impairment were related to frequency of explosive anger episodes. Explosive anger was associated with age ( $>35$ years), being married, low levels of education, being employed, traumatic event count, ongoing adversity and intimate partner violence.

\section{Conclusions}

A combination of social programmes and novel psychological therapies may assist in reducing severe anger among pregnant and post-partum women in conflict-affected countries such as Timor-Leste.

\section{Declaration of interest}

None.

\section{Copyright and usage}

(c) The Royal College of Psychiatrists 2015. This is an open access article distributed under the terms of the Creative Commons Non-Commercial, No Derivatives (CC BY-NC-ND) licence.
Depression among women in pregnancy and the post-partum period has attracted substantial research attention ${ }^{1-3}$ but there has been less of a focus on other affective responses such as explosive anger. There is some evidence from population-wide studies that explosive anger may represent an important response among the generality of women living in conflictaffected, low-income societies. ${ }^{4,5}$ For example, a survey of two villages in Timor-Leste found that two out of five women reported experiencing one or more anger attacks a month and $12 \%$ met criteria for DSM-IV-defined intermittent explosive disorder. ${ }^{4,6}$ Explosive anger was associated with exposure to the traumatic events of conflict, ongoing conditions of adversity and intimate partner violence among these women., ${ }^{4,7}$ In qualitative interviews, women commonly reported onset or worsening of explosive anger during the period of pregnancy and the post-partum. ${ }^{4,7} \mathrm{We}$ acknowledge the risk of stigmatising women in post-conflict settings by focusing on expressions of anger and aggression. Our aim, however, is to reveal the historical and social factors underpinning expressions of women's anger with the explicit objective of informing policy and practices that advance the status, opportunities and living conditions of women in post-conflict environments. The aims of the study are to document the prevalence of explosive anger among Timorese women in the mid-trimester of pregnancy and 3-6 months post-partum. We test the hypotheses that greater frequency of explosive anger episodes will be associated with higher levels of functional impairment; and that explosive anger will be related to a constellation of past and ongoing adversities, including exposure to the traumatic events of conflict, intimate partner violence and conditions of poverty and insecurity.

\section{Method}

We report the study in accordance with the Strengthening the Reporting of Observational Studies in Epidemiology (STROBE) guidelines.

\section{Context and historical background}

Timor-Leste is a half-island nation situated to the north of Australia and the east of the main Indonesian archipelago. The indigenous population experienced extensive human rights violations including murder, torture, arbitrary incarceration, mass displacement and extreme deprivations during the prolonged Indonesian occupation (1975-1999). ${ }^{8}$ In 1999, following a popular vote in favour of national independence, militia supported by the Indonesian military initiated a campaign of violence which led to the destruction of infrastructure, widespread burning of houses and mass displacement of the population. ${ }^{9}$ United Nations intervention restored conditions of peace that largely were maintained through the period of independence (2002). In 2006-2007, there was a further outbreak of conflict involving warring factions within the country. ${ }^{10}$ Again, there was mass displacement of populations, injuries, deaths and extreme difficulties accessing food, water and health services.

\section{Study setting}

We conducted the study between June 2012 and June 2013 in Aileu and Liquiça, two adjacent districts of the nine that comprise Timor-Leste. Two rural and two urban administrative units (sucos) in Aileu District and one each in Liquiça were included, the population size per suco being larger at the latter site. The districts include areas that are broadly typical of the country as a 
whole, including mountainous and coastal as well as rural and urban locations. Our choice of sites was partly influenced by access (in a country where the road system remains poor) and the established relationships with local services of our partner agency, the Alola Foundation, the peak women's non-governmental organisation (NGO) in Timor-Leste.

\section{Sample}

Our sampling frame was designed to identify all women in the mid-trimester of pregnancy and 3-6 months post-partum residing in the defined administrative units, using all available methods of identification to ensure that as few eligible women as possible were overlooked. We selected the mid-trimester when physical evidence of pregnancy is obvious in a setting where access to selftesting in the earlier phase of gestation is not widely available. A further reason for selecting the designated periods of pregnancy and the post-partum was to reduce the likelihood of recording transient forms of emotional upheaval surrounding the immediate period of childbirth. Exclusion criteria were the presence of acute psychosis, cognitive or intellectual impairment that interfered with women's capacity to comprehend the interview, and the presence of severe medical illness.

Our aim was to include all eligible women residing in the study locations, using a comprehensive, step-wise approach in order to maximise identification. One hundred and seventy-four women ( $40.7 \%$ of the final sample) were identified from registers held by perinatal community services, 51 (11.9\%) from general community health service registers, 109 (25.5\%) based on the reports of chefes (chiefs), who are knowledgeable about the community as a whole, and 93 additional women (21.8\%), not already detected by the preceding strategies, during a final, comprehensive, door-to-door survey of all households in the designated geographical areas.

\section{Interview procedure}

All women identified were approached; three declined to participate and one discontinued the interview, yielding a $>99 \%$ response rate of eligible women identified. Most women were interviewed at clinics, although a minority chose to be visited at their homes.

We followed our previous practice, based on wide consultation, by providing participants with a package of soap to compensate for their time.

\section{Data management}

Paper and pencil surveys were checked immediately following interview and again at the research office prior to being entered in de-identified format by a trained data assistant into a password protected computer. All electronic data files were checked by an independent member of the team in order to detect missing data, out-of-range values and logically incompatible responses. Where potential errors were identified, electronic data were corrected with reference to original paper survey records. Original surveys were stored in a locked cabinet in the research office prior to being transferred to the University of New South Wales in Sydney for secure storage.

\section{Survey measures}

Socio-demographic data were based on items from the national census. Among questions concerning the pregnancy, we inquired whether the woman had planned and/or wanted to have the child.

We applied internationally recognised guidelines to adapt, translate and back-translate measures of explosive anger, posttraumatic stress disorder and general distress. ${ }^{4,11,12}$

\section{Explosive anger}

In our past population-wide studies, we used qualitative and quantitative procedures to develop our community measure of explosive anger. ${ }^{12}$ The initial questions were based on the DSM-IV criteria for intermittent explosive disorder, including items relating to aggression directed at persons or property. In an iterative process of qualitative field testing and feedback, we modified the expression and formatting of questions to ensure their linguistic, idiomatic and semantic appropriateness within the culture. A key focus was to ensure ease of administration and comprehension of the anger module in a setting of low literacy and education.

In a community-based study, we compared our field measure with intermittent explosive disorder assignments on the structured clinical interview (SCID) for DSM-IV, administered in a blinded manner by Australian psychologists familiar with the Timorese culture. ${ }^{13}$ There was a high level of convergence across the two measures: area under the curve (AUC) 0.90 (95\% CI 0.83-0.98); sensitivity $93.3 \%$; specificity $87.5 \%$; positive predictive power 0.89 ; negative predictive power 0.92 ; and overall correct classification $90.6 \%$. Given ongoing uncertainties about the transcultural status of intermittent explosive disorder as a nosological category, we apply the term explosive anger herein.

In our statistical analyses, we applied two indices of anger, the category of explosive anger (defined by intermittent explosive disorder criteria) and the frequency of explosive anger episodes applied across the sample as a whole. Our frequency classification was based on our prior knowledge that many Timorese are not calendar-focused, limiting the extent to which fine-grained timelines can be provided by participants in relation to onset, duration and frequency of symptoms. We therefore developed a hierarchy of broadly defined, mutually exclusive categories, according to the minimum number of episodes experienced per unit of time. We generated four levels: (1) at the highest level of frequency, respondents needed to experience a minimum of one episode of explosive anger a day (over several days), although the frequency could be greater; (2) at the next level, a minimum of one episode a week for several weeks (but below the threshold of level 1); (3) a minimum of one episode a month for several months (but not reaching threshold for categories 1 and 2); and (4) the remainder reported no explosive anger or only rare episodes.

\section{Post-traumatic stress disorder (PTSD)}

The Harvard Trauma Questionnaire (HTQ) assesses 16 symptoms of PTSD, each scored on a 4-point frequency scale. The summary score (1-4) is calculated by dividing the total score by the number of items. ${ }^{15}$ Our aforementioned convergence study showed a sound level of agreement between the HTQ symptom scale and the SCID for DSM-IV PTSD module applied by psychologists: AUC $=0.82 \quad(95 \%$ CI $0.71-0.94){ }^{13}$ In the present sample, Cronbach's alpha for the item pool was $0.93 .^{16}$ We applied a community threshold score of $\geq 2.0$ to identify significant symptoms of PTSD in this sample. ${ }^{17}$

\section{Psychological distress}

The Kessler-10 (K10) psychological distress measure consists of 10 items each scored on a five-point frequency scale. Most symptoms assess depression although items for anxiety and somatic symptoms are included. ${ }^{13}$ The K10 has been used extensively across cultures as a proxy measure for depression in the perinatal period. ${ }^{19}$ The international threshold score of $>30$ showed a sound level of agreement with the SCID module for major depressive disorder in the aforementioned convergence study. ${ }^{18}$ Cronbach's alpha for the 10 items in the present data set was 0.91 . 


\section{Functional impairment}

Functional impairment was assessed using the abbreviated version of the WHO Disability Assessment Schedule (WHODAS 2.0, 12-item version), based on the International Classification of Functioning and Health. ${ }^{6}$ Six core domains are assessed including cognition/communication, mobility, self-care, interpersonal interaction, life activities and participation in society. Items are rated on a 5 -point Likert scale: none $=1$, mild $=2$, moderate $=3$, severe $=4$ and extreme $=5$. The measure has been used extensively across cultures including among samples of women in pregnancy. ${ }^{20,21}$ The WHODAS showed a moderate level of convergence with a locally developed index of functioning among a sample of pregnant women living in deprived conditions in South Africa. ${ }^{21}$ Internal reliability (Cronbach's alpha) for the total pool of items in our data set was 0.89 . We derived a mean score for the total scale and each subdomain of functional impairment.

\section{Traumatic events}

We previously modified the traumatic event list of the HTQ to ensure its relevance to Timor-Leste. ${ }^{12}$ The list principally comprises conflict-related traumatic events (exposure to torture, combat, conflict-related injury, witnessing murder and extreme deprivation arising from fire, loss of shelter and lack of food and water). Additional items assessed disasters (flood and earthquake) which are common in Timor-Leste. We repeated the list of traumatic events for two historical periods, the Indonesian occupation and humanitarian emergency; and the subsequent period (>1999) which included the internal conflict of 2006-2007. A summary traumatic event count was generated for each historical period based on assigning a score of 1 for each type of traumatic event endorsed.

\section{Intimate partner violence}

The WHO Multi-Country Study on Women's Health and Domestic Violence measure has been used in a wide range of countries worldwide. ${ }^{22}$ In a study undertaken in Brazil, confirmatory factor analysis supported the three-factor structure (physical, sexual and emotional abuse) of the measure. In our analysis, we applied the 12-month prevalence of physical intimate partner violence enacted by the current or most recent partner.

\section{Ongoing adversity}

In a previous community-based study, we developed an index of ongoing adversity relevant to contemporary life in Timor-Leste based on an iterative process of qualitative interviews, field testing and feedback by a Timorese consultative group that was diverse in age, gender and education. ${ }^{22}$ Piloting in preparation for the present study identified 11 items from the larger list that were specifically relevant to women in pregnancy and the post-partum. Items assessed aspects of poverty (lack of clean water and food, insufficient money for school fees and to meet traditional obligations to family (lia), poor shelter and unemployment) and insecurity (conflict with spouse, children, extended family, youth and the wider community). A total adversity score ranging from 0 to 11 was generated by the addition of items (assigned 1 if rated $\geq 2$ on a four-point severity scale). Based on the distribution of scores we created four intervals: $0-4$, a minimal problem; $5-7$, a problem; $8-10$, a moderately serious problem; $\geq 11$, a very serious problem.

\section{Ethics}

The Human Research Ethics Committee of the University of New South Wales and the National Health Research Cabinet of TimorLeste approved the study. Women were informed verbally of the nature of the study because of low literacy levels. They either signed or marked translated consent forms in the presence of a witness.

\section{Statistical analyses}

We present descriptive statistics for traumatic event exposure, intimate partner violence, ongoing adversity, explosive anger and other mental health indices. Patterns of comorbidity were examined using tetrachoric correlation coefficients. To limit the number of categories in multivariate analysis, women with no schooling and primary schooling were aggregated after bivariate analysis showed no difference between the two groups in the prevalence of explosive anger. Consistent with past studies in Timor-Leste, only a small number of respondents $(n=41)$ reported that they had not been exposed to any of the listed traumatic events. Preliminary analyses showed that those reporting 0 and 1 traumatic events did not differ in their rates of explosive anger, allowing us to aggregate these women to form the reference group (0-1 traumatic events) in multivariate comparisons with those experiencing $\geq 2$ traumatic events.

Results of the bivariate analyses are represented as percentages and chi-square tests. In our multivariate logistic regression analysis, we examined for associations of socio-demographic factors, traumatic event count, intimate partner violence and adversity with the category of explosive anger, controlling for clustering by district and other mental health indices (threshold assignment to PTSD, psychological distress). We represent the findings as adjusted odds ratios (AORs) with 95\% confidence intervals (95\% CIs).

For four participants, single missing values (one for each respondent) on individual mental health indices were replaced with the respondent's mean score for the remaining items on the relevant measure. Analyses were performed using IBM SPSS version 22 for windows.

\section{Results}

\section{Sample characteristics}

Of the 427 participants, 257 were in the second trimester of pregnancy and 170 were 3-6 months post-partum. Table 1 indicates that results for pregnant and post-partum women were similar for most indices measured, including the prevalence of explosive anger. Post-partum women reported greater exposure to traumatic events in the pre-1999 period, most likely reflecting the slightly higher number of older and lower number of younger women in that group (noting that in both subsamples, most women were aged 20-34 years).

The majority were married; $11.5 \%$ were living in cohabiting intimate relationships (in Timor-Leste, a common prelude to marriage). Half had completed high school, a small percentage also receiving post-school education or training. More than twothirds of women were fully occupied with home duties (69.6\%), the remainder engaged in part-time or full-time work in farming, fishing, small trade or the government sector.

\section{Prevalence of conflict-related traumatic events, ongoing adversity and intimate partner violence}

The majority of women (62.5\%) reported exposure to two or more traumatic events. The pattern of traumatic events reported was consistent with the history of the country and the types of events that a woman in the specified age range would have experienced as children, adolescents or young adults. For example, more women reported exposure to situations of combat/warfare for the pre-1999 Indonesian period ( $28 \% v .1 \%$ for the post-1999 period), having their house burnt down, a common occurrence in the 
Table 1 sociodemographic and mental health characteristics of Timorese women in the second trimester and 3-6 months post-partum

\begin{tabular}{|c|c|c|c|c|c|c|}
\hline \multirow[b]{2}{*}{ Sociodemographic characteristics and mental health indices } & \multicolumn{2}{|c|}{ Pregnant } & \multicolumn{2}{|c|}{ Post-partum } & \multicolumn{2}{|c|}{ Whole sample } \\
\hline & $n$ & $\%$ & $n$ & $\%$ & $n$ & $\%$ \\
\hline & 257 & 100.0 & 170 & 100.0 & 427 & 100.0 \\
\hline \multicolumn{7}{|l|}{ Age (years) } \\
\hline$\leq 20$ & 30 & 11.7 & 16 & 9.4 & 46 & 10.8 \\
\hline $20-24$ & 87 & 33.9 & 58 & 34.1 & 145 & 34.0 \\
\hline $25-34$ & 122 & 47.5 & 77 & 45.3 & 199 & 46.6 \\
\hline$\geq 35$ & 18 & 7.0 & 19 & 11.2 & 37 & 8.7 \\
\hline Mean age (s.d.) & \multicolumn{2}{|c|}{$25.6(5.7)$} & \multicolumn{2}{|c|}{$26.5(6.2)$} & \multicolumn{2}{|c|}{$26.0(5.9)$} \\
\hline Median & \multicolumn{2}{|c|}{25.0} & \multicolumn{2}{|c|}{25.0} & \multicolumn{2}{|c|}{25.0} \\
\hline \multicolumn{7}{|l|}{ Marital status ${ }^{1}$} \\
\hline Married & 221 & 86.0 & 157 & 92.4 & 378 & 88.5 \\
\hline Living together & 36 & 14.0 & 13 & 7.6 & 49 & 11.5 \\
\hline \multicolumn{7}{|l|}{ Highest level of completed education } \\
\hline None & 30 & 11.7 & 31 & 18.2 & 61 & 14.3 \\
\hline Primary school & 83 & 32.3 & 50 & 29.4 & 133 & 31.1 \\
\hline Junior or senior high school & 131 & 51.0 & 82 & 48.2 & 213 & 49.9 \\
\hline Technical diploma or university & 13 & 5.1 & 7 & 4.1 & 20 & 4.7 \\
\hline \multicolumn{7}{|l|}{ Occupation } \\
\hline Unemployed & 170 & 66.1 & 127 & 74.7 & 297 & 69.6 \\
\hline Government or small trade & 36 & 14 & 21 & 12.4 & 57 & 13.3 \\
\hline Others (fishing, farming etc.) & 51 & 19.8 & 22 & 12.9 & 73 & 17.1 \\
\hline \multicolumn{7}{|l|}{ Conflict-related traumatic events (prior to 1999)2 } \\
\hline None or one & 112 & 43.6 & 58 & 34.1 & 170 & 39.8 \\
\hline Two or more & 145 & 56.4 & 112 & 65.9 & 257 & 60.2 \\
\hline \multicolumn{7}{|l|}{ Conflict-related traumatic events (since 1999) } \\
\hline None or one & 98 & 38.1 & 62 & 36.5 & 160 & 37.5 \\
\hline Two or more & 159 & 61.9 & 108 & 63.5 & 267 & 62.5 \\
\hline \multicolumn{7}{|l|}{ Ongoing adversity ${ }^{3}$} \\
\hline A minimal problem & 50 & 19.5 & 25 & 14.7 & 75 & 17.6 \\
\hline A problem & 81 & 31.5 & 50 & 29.4 & 131 & 30.7 \\
\hline A moderately serious problem & 78 & 30.4 & 54 & 31.8 & 132 & 30.9 \\
\hline A very serious problem & 48 & 18.7 & 41 & 24.1 & 89 & 20.8 \\
\hline \multicolumn{7}{|l|}{ Intimate partner violence (physical intimate partner violence) ${ }^{4}$} \\
\hline No & 179 & 69.6 & 126 & 74.1 & 305 & 71.4 \\
\hline Yes & 78 & 30.4 & 44 & 25.9 & 122 & 28.6 \\
\hline \multicolumn{7}{|l|}{ Severe psychological distress ${ }^{5}$} \\
\hline No & 244 & 94.9 & 159 & 93.5 & 403 & 94.4 \\
\hline Yes & 13 & 5.1 & 11 & 6.5 & 24 & 5.6 \\
\hline \multicolumn{7}{|l|}{$\mathrm{PTSD}^{6}$} \\
\hline No & 238 & 92.6 & 149 & 87.6 & 387 & 90.6 \\
\hline Yes & 19 & 7.4 & 21 & 12.4 & 40 & 9.4 \\
\hline \multicolumn{7}{|l|}{ Explosive anger $^{7}$} \\
\hline No & 144 & 56.0 & 97 & 57.1 & 241 & 56.4 \\
\hline Yes & 113 & 44.0 & 73 & 42.9 & 186 & 43.6 \\
\hline Functional impairment ${ }^{8}$ & & & & & & \\
\hline No & 137 & 53.3 & 82 & 48.2 & 219 & 51.3 \\
\hline Yes & 120 & 46.7 & 88 & 51.8 & 208 & 48.7 \\
\hline $\begin{array}{l}\text { 1. Pregnant women differed significantly from post-partum women in } \\
\text { 2. Conflict-related traumatic events before } 1999 \text { include events during } t \\
\text { include the period of internal conflict of } 2000-2007 \text {. In both periods, eve } \\
\text { fire, lack of shelter, and insufficieient food and water, with a few items } \\
\text { 3. Based on whether at least one item of ongoing adversity was rated } \\
\text { 4. Based on current or most recent partner. } \\
\text { 5. Based on locally validated threshold score for severe distress of } \geq 3 \\
\text { 6. PTSD cases were determined using a locally validated cut-off of } 2.0 \\
\text { 7. Cases of explosive anger were identififed using DSM-IV intermittent } \\
\text { 8. The threshold for functional impairment was based on a score of } 2\end{array}$ & $\begin{array}{l}\text { tatus } \\
\text { exian } \\
\text { de hum } \\
\text { to natu } \\
\text { lal prob } \\
\text { on the } \\
\text { o disor } \\
\text { ater, th }\end{array}$ & $\begin{array}{l}\text { and subse } \\
\text { ated traun } \\
\text { i ther tra } \\
\text { problem } \\
\text { symptom } \\
\text { re derive }\end{array}$ & $\begin{array}{l}\text { nanitariar } \\
\text { ing murc } \\
\text { ide child } \\
\text { oderatel }\end{array}$ & $\begin{array}{l}\text { cy; conflic } \\
\text { yor others } \\
\text { encec rela } \\
\text { roblem (8 }\end{array}$ & $\begin{array}{l}\text { aumatic } \\
\text { eprivation } \\
\text { tic even } \\
\text { ery seri }\end{array}$ & $\begin{array}{l}\text { ce } 1999 \\
\text { care, } \\
\text { mily. } \\
\text { m ( } \geq 11) .\end{array}$ \\
\hline
\end{tabular}

emergency of 1999 (33.5\% v. 3.7\%), and experiences of family persecution arising from links to the armed resistance movement (57\% v. 11\%).

In relation to ongoing adversity, four out of five women reported experiencing at least one of the listed stressors as a serious problem in the previous 12 months. In addition, almost a third of women $(28.6 \%)$ reported experiencing physical intimate partner violence enacted by the current or most recent partner in the past 12 months.

\section{Prevalence of explosive anger, PTSD and psychological distress}

Over two-fifths of women $(186,43.6 \%)$ met criteria for the category of explosive anger. One in $20(5.1 \%)$ reached the 


\begin{tabular}{|c|c|c|c|}
\hline Severe distress & Severe distress ${ }^{1}$ & $\mathrm{PTSD}^{2}$ & Explosive anger ${ }^{3}$ \\
\hline Severe distress & 1.00 & & \\
\hline PTSD & $0.78^{\star \star}$ & 1.00 & \\
\hline Explosive anger & $0.51^{* *}$ & $0.45^{\star}$ & 1.00 \\
\hline \multicolumn{4}{|c|}{$\begin{array}{l}\text { 1. Severe distress based on a score of } \geq 30 \text { on } \mathrm{K} 10 \text {. } \\
\text { 2. PTSD using a cut-off point of } 2.0 \text { based on mean PTSD symptom scores. } \\
\text { 3. Explosive anger defined by DSM-IV intermittent explosive disorder criteria. } \\
{ }^{*} P<0.05 ;{ }^{* *} P<0.01 \text {. }\end{array}$} \\
\hline
\end{tabular}

threshold for severe psychological distress on the K10 (score $\geq 30$ ) and $7.4 \%$ met a pre-established community threshold for PTSD $(>2.0)$. Explosive anger was moderately correlated with the other mental health indices: (PTSD 0.45, $P<0.01 ; \mathrm{K} 10$ 0.51, $P<0.01$; Table 2).

\section{Functional impairment}

Women with explosive anger scored statistically higher on all individual WHODAS items (all $P<0.05$ ) with the exception of difficulties in dressing and walking. There was a dose-effect relationship between the frequency of explosive anger episodes and the WHODAS total as well as all subdomain scores except self-care (Table 3). The relationship remained consistent whether or not other mental health indices (K10 and PTSD) were entered as covariates in the analysis (data available on request).

\section{Trauma, ongoing adversity and explosive anger}

Whether the pregnancy was planned or wanted showed no association with either intimate partner violence or explosive anger in bivariate analyses.

In multivariate analysis, socio-demographic variables associated with explosive anger included age (particularly women $>35$ years), being married, having lower levels of education and working in government employment (Table 4). In relation to substantive variables, the traumatic event count for the Indonesian period was associated with explosive anger, the post-1999 traumatic event count (significant in bivariate analysis) no longer contributing because of collinearity with the traumatic event index of the earlier period. There was a strong, dose-effect relationship between ongoing adversity and explosive anger. In addition, women experiencing intimate partner violence had higher rates of explosive anger.

\section{Discussion}

Two of five women in our sample of Timorese women in the second trimester of pregnancy and 3-6 months post-partum were assigned to the category of explosive anger. Across the sample as a whole, there was a dose-effect relationship between the frequency of explosive anger episodes (ranging from a minimum of one episode a day to one a month) and a measure of functional impairment. As hypothesised, in multivariate analysis, explosive anger was associated with the traumatic events of mass conflict, particularly those experienced during the Indonesian period, ongoing adversity related to poverty and insecurity, and exposure to physical intimate partner violence.

\section{Strengths and limitations of study}

Prior to exploring the implications of our findings, we consider the strengths and limitations of our study. To our knowledge, the study is the first worldwide to investigate the occurrence of explosive anger among women in pregnancy and the post-partum in a low-income, post-conflict country. We used a comprehensive recruitment strategy to identify eligible women in the designated geographical areas and the response rate of women approached was very high. Our study sites included urban and rural as well as mountainous and coastal areas, typical of the geographical and social structure of the country as a whole. We cannot determine, however, whether our selection of study sites introduced bias into our data. Future studies will be needed among more representative samples of pregnant and post-partum women in Timor-Leste and in other conflict-affected countries to test the generalisability of our findings. Larger studies will be able to investigate factors relevant to explosive anger that are specific to various stages of pregnancy and the postnatal period. ${ }^{1}$ Longitudinal studies also may allow closer delineation of the temporal sequencing of variables, for example, whether women's anger follows first exposure to intimate partner violence or vice versa, although accurate assessment of the precise chronology of interpersonal interactions within spouse dyads remains a challenge.

International measures (such as the HTQ, K10 and WHODAS) facilitate cross-country comparisons but may be insensitive to culture-specific manifestations of distress and functional impairment. We adapted our measure of anger to the study context based on an iterative process of qualitative assessment and feedback. All symptom measures showed sound convergence with a clinical diagnosis made by trained psychologists familiar with the context. The WHODAS has been applied among pregnant and post-partum women in different cultures, the measure showing a moderate level of convergence with a contextually developed index of functioning in a recent study among pregnant women in South Africa. ${ }^{21}$ We note that our analysis did not focus primarily on deriving an absolute cut-off for functional impairment but rather on assessing within-group differences in relation to increasing frequency of explosive anger episodes. The regular dose-effect pattern we found suggests that the WHODAS performed effectively in making the hypothesised distinctions in this context.

Table 3 Associations of frequency of explosive anger episodes and functioning according to the WHODAS and its subdomains

\begin{tabular}{|c|c|c|c|c|c|c|c|}
\hline \multirow[b]{2}{*}{ Episodes of anger attacks ${ }^{1}$} & \multicolumn{7}{|c|}{ WHODAS subdomains } \\
\hline & Cognition & Mobility & Self-care & Getting along & Life activities & $\begin{array}{c}\text { Community } \\
\text { participation }\end{array}$ & $\begin{array}{l}\text { Total } \\
\text { score }\end{array}$ \\
\hline No/only occasional episodes $(n=88)$ & 3.2 & 4.5 & 2.1 & 2.6 & 3.7 & 3.6 & 19.7 \\
\hline Minimum once a month $(n=158)$ & 4.1 & 4.5 & 2.3 & 3.0 & 4.1 & 4.3 & 22.3 \\
\hline Minimum once a week $(n=123)$ & 5.0 & 5.2 & 2.4 & 4.6 & 5.1 & 5.1 & 27.4 \\
\hline Minimum once a day $(n=58)$ & 5.1 & 6.0 & 2.2 & 5.0 & 5.6 & 5.2 & 29.1 \\
\hline$P$ (linearity test) & $<0.01$ & $<0.01$ & 0.28 & $<0.01$ & $<0.01$ & $<0.01$ & $<0.01$ \\
\hline
\end{tabular}




\begin{tabular}{|c|c|c|c|c|c|}
\hline & \multicolumn{5}{|c|}{ Explosive Anger $^{1}$} \\
\hline & & Total, $n$ & $\%$ & Unadjusted odds ratio (95\% CI) & Adjusted odds ratio $(95 \% \mathrm{Cl})$ \\
\hline All women & 427 & 186 & 43.6 & & \\
\hline 3-6 months pregnant & 257 & 113 & 44.0 & 1 [Reference] & 1 [Reference] \\
\hline 3-6 months post-partum & 170 & 73 & 42.9 & $0.96(0.65-1.42)$ & $0.78(0.50-1.23)$ \\
\hline \multicolumn{6}{|l|}{ Age (years) } \\
\hline$\leq 20$ & 46 & 16 & 34.8 & 1 [Reference] & 1 [Reference] \\
\hline $20-24$ & 145 & 49 & 33.8 & $0.96(0.48-1.92)$ & $0.97(0.49-2.28)$ \\
\hline $25-34$ & 199 & 92 & 46.2 & $1.61(0.83-3.14)$ & $1.61(0.77-3.39)$ \\
\hline$\geq 35$ & 37 & 29 & 78.4 & $6.80(2.52-18.30)^{* *}$ & $6.08(2.0-18.49)^{\star *}$ \\
\hline \multicolumn{6}{|l|}{ Marital status } \\
\hline Married & 378 & 177 & 46.8 & 1 [Reference] & 1 [Reference] \\
\hline Living together & 49 & 9 & 18.4 & $0.26(0.12-0.54)^{\star *}$ & $0.33(0.15-0.75)^{*}$ \\
\hline \multicolumn{6}{|l|}{ Education } \\
\hline None or primary & 194 & 103 & 53.1 & 1 [Reference] & 1 [Reference] \\
\hline High school or post-school & 233 & 83 & 35.6 & $0.49(0.3-0.72)^{\star *}$ & $0.60(0.38-0.94)^{\star}$ \\
\hline \multicolumn{6}{|l|}{ Occupation } \\
\hline Unemployed & 297 & 126 & 42.4 & 1 [Reference] & 1 [Reference] \\
\hline Government or small trade & 57 & 34 & 59.6 & $2.01(1.13-3.57)^{\star}$ & $2.20(1.11-4.37)^{*}$ \\
\hline Other & 73 & 26 & 35.6 & $0.75(0.44-1.28)$ & $0.74(0.40-1.39)$ \\
\hline \multicolumn{6}{|c|}{ Conflict-related traumatic events (prior to 1999) } \\
\hline None or one & 170 & 55 & 32.4 & 1 [Reference] & 1 [Reference] \\
\hline Two or more & 257 & 131 & 51.0 & $2.17(1.45-3.26)^{\star *}$ & $1.70(1.07-2.71)^{*}$ \\
\hline \multicolumn{6}{|c|}{ Conflict-related traumatic events (since 1999) ${ }^{2}$} \\
\hline None or one & 160 & 53 & 33.1 & 1 [Reference] & \\
\hline Two or more & 267 & 133 & 49.8 & $2.00(1.3-3.01)^{\star}$ & \\
\hline \multicolumn{6}{|l|}{ Ongoing adversity } \\
\hline A minimal problem & 75 & 11 & 14.7 & 1 [Reference] & 1 [Reference] \\
\hline A problem & 131 & 59 & 45.0 & $4.77(2.31-9.86)^{\star \star *}$ & $4.44(2.01-9.83)^{\star \star *}$ \\
\hline A moderately serious problem & 132 & 67 & 50.8 & $6.00(2.90-12.38)^{\star *}$ & $5.17(2.32-11.51)^{\star *}$ \\
\hline A very serious problem & 89 & 49 & 55.1 & $7.13(3.32-15.30)^{* *}$ & $5.34(2.24-12.74)^{* \star}$ \\
\hline \multicolumn{6}{|l|}{ Intimate partner violence } \\
\hline No & 305 & 115 & 37.7 & 1 [Reference] & 1 [Reference] \\
\hline Yes & 122 & 71 & 58.2 & $2.30(1.50-3.53)^{* *}$ & $1.88(1.15-3.07)^{* *}$ \\
\hline \multicolumn{6}{|c|}{$\begin{array}{l}\text { 1. Explosive anger based on DSM-IV intermittent explosive disorder criteria. } \\
\text { 2. The traumatic event count since } 1999 \text { was excluded from the multivariate analysis due to a high level of collinearity with the pre-1999 traumatic event count. Multivariate analysis } \\
\text { controlled for clustering by district (Liquiça, Aileu), PTSD (score }>2.0 \text { ) and severe psychological distress (K10 score } \geq 30 \text { ). AORS are presented with } 95 \% \text { Cl. } \\
\star P<0.05, * * P<0.01 \text {. }\end{array}$} \\
\hline
\end{tabular}

The cross-sectional design of the study cautions against drawing causal inferences. Although retrospective errors in reporting could influence the accuracy of recording of traumatic events, the pattern of responses obtained was consistent with the known history of Timor-Leste and with the types of events that would have affected female children and adolescents as well as young women during the periods of conflict. We also note that any bias arising from the forgetting of conflict-related traumatic events that occurred in early childhood would have attenuated rather than exaggerated the relationship we found between our traumatic event count and explosive anger.

\section{Independence of the construct of explosive anger}

An important question is the extent to which explosive anger is independent of the diagnosis of PTSD given that the latter category incorporates symptoms of irritability and anger. Although the disparity in prevalence between the two categories (explosive anger and PTSD) suggests their relative independence as constructs, that discrepancy may be attributable to variation in cut-off thresholds applied in this cross-cultural context. In that respect, we note that the cut-off applied for PTSD was based on a community study undertaken in another setting. Nevertheless, our multivariate analysis controlling for PTSD (as well as severe psychological distress) indicated an independent relationship between explosive anger and a range of relevant historical and social factors, including past exposure to traumatic events, ongoing adversity and intimate partner violence, findings that support the construct validity of our anger index.

\section{Potential origins of explosive anger}

The finding that two out of every five women in the designated periods of pregnancy and the post-partum met criteria for explosive anger requires careful interpretation. In our past general population study in Timor-Leste in which we used the same measure, $12 \%$ of women met criteria for explosive anger based on intermittent explosive disorder criteria. ${ }^{4}$ In nationally representative studies conducted in other countries, the prevalence of intermittent explosive disorder has varied from $1.5 \%$ in Iraq to $7.3 \%$ in the USA,${ }^{23-25}$ noting that comparisons should be made with caution given that in the latter studies, a different diagnostic measure was used.

It is possible that pregnancy and the post-partum are high-risk periods for explosive anger. A study undertaken among a clinic population in the USA found $60 \%$ of women in the same periods of pregnancy and the post-partum met criteria for anger attacks, more broadly defined than the index we used in the present study. ${ }^{25}$ Further studies are needed, therefore, to examine the universal factors, particularly related to the biological changes that 
occur in pregnancy and the post-partum, that may contribute to expressions of explosive anger. For example, there is evidence that androgen levels increase in pregnancy and one component, testosterone, is known to be associated with expressions of anger and aggression. ${ }^{26}$

\section{Anger as a normative response}

Like other emotions, anger varies on a spectrum from the normative to the pathological. Defining the cut-off between culturally acceptable expressions of anger and morbid manifestations in the present context presents a challenge, particularly given the long history of adversity that Timorese women had experienced. The conflict-related traumatic events experienced during the Indonesian occupation occurred a decade or more prior to our study, yet these events continued to contribute to levels of explosive anger. In addition, almost a third of women experienced physical intimate partner violence in the prior year, a factor that was independently associated with explosive anger. Finally, a strong and stepwise association was found between conditions of ongoing adversity and explosive anger, reinforcing past evidence that poverty and insecurity contribute substantially to levels of distress among women in post-conflict environments. ${ }^{4}$ Given this background, among many of the women participating in our study, explosive anger may be regarded as representing a legitimate and adaptive response to cumulative experiences of adversity extending over a protracted period of their lives.

\section{Pathological forms of anger}

At the same time, it is important to recognise that severe forms of anger may be a source of distress and functional impairment among some Timorese women. One in eight women participating in our survey experienced one or more episodes of explosive anger a day and these women reported the highest level of functional impairment relative to their peers. Women participating in our previous community study reported wide-ranging difficulties in functioning associated with explosive anger which they regarded as a health crisis requiring medical attention. ${ }^{4}$ In addition, mothers with explosive anger commonly reported that accompanying acts of aggression were directed at their children, on occasions leading to severe physical harm. ${ }^{4}$ These accounts suggest that in families where there is a convergence of high exposure to the traumatic events of conflict, ongoing intimate partner violence and severe ongoing adversity, maternal anger can pose risks to the future mental health of offspring, a transgenerational pathway that warrants further examination. ${ }^{27}$

\section{Policy, clinical and research implications}

Our findings point to the imperative of promoting the status, rights and protection of women residing in low-income, postconflict countries such as Timor-Leste, a key focus being on women in the reproductive period. Developing strategies to combat intimate partner violence must be a high priority. In Timor-Leste, legislation has been adopted to criminalise intimate partner violence and prosecutions against perpetrators have increased in recent years. In addition, services to protect women have been established. Nevertheless, as in other countries, much further work is needed to achieve durable changes to entrenched patriarchal attitudes and gender-related power imbalances that underlie the perpetuation and condoning of intimate partner violence. $^{28}$ In addition, women in these settings often are burdened with providing for the basic needs and care of children, a task that may become even more onerous in pregnancy and the post-partum period. Overcoming poverty and enabling women to achieve economic independence are vital to relieving this burden.
Providing psychological support for the generality of women experiencing explosive anger is beyond the capacity of core mental health services in countries such as Timor-Leste. At best, mental health services may be able to intervene for the minority of women who are most severely disabled by explosive anger and comorbid disorders. As yet, no clear guidelines can be offered to direct psychotherapeutic interventions. Although there is preliminary evidence that psychological treatments for explosive anger can be effective among refugees in high-income countries, ${ }^{29}$ no comparable studies have been undertaken in low-income countries. In trialling interventions, a focus on combined family therapy and social interventions may be appropriate, particularly given that ongoing family stressors and intimate partner violence appear to contribute substantially to maternal explosive anger.

Finally, our findings suggest the importance of conducting further research into the phenomenon of explosive anger among pregnant and post-partum women across a range of low-income, post-conflict societies worldwide. Future studies should examine the impacts of explosive anger on the longer-term mental and physical health of women, and the design and testing of effective interventions to prevent and reduce this burden. Also of importance is furthering knowledge on the impacts of explosive anger on parenting styles and the consequent impact on the development and longer-term psychosocial well-being of offspring affected by this maternal response pattern. ${ }^{27}$ The overall objective of this direction of research should be to promote the mental health of women in the reproductive period and to afford them maximal opportunities (in their family roles, in work and in society as a whole) to participate in the process of post-conflict recovery.

\section{Funding}

The study was funded by an Australian National Health and Medical Research Council Grant.

\section{Acknowledgements}

The Alola Women's Foundation of Timor-Leste for their advice and support for this project and our team.

Derrick Silove, MB ChB, FRANZCP, MD, Professor and Director, Psychiatry Research and Teaching Unit and Ingham Institute, University of New South Wales, Sydney, Australia; Susan Rees, PhD, MSocPol, Senior Lecturer, Psychiatry Research and Teaching Unit, University of New South Wales and Ingham Institute, Liverpool Hospital, Sydney, Australia; Natalino Tam, In Country Project Manager, Psychiatry Research and Teaching Unit, University of New South Wales, Sydney, Australia; Mohammed Mohsin, PhD, Senior Research Officer, Psychiatry Research and Teaching Unit, Southwest Sydney Local Health District, Sydney, Australia; Alvin Kuowei Tay, MForPsych, Senior Research Officer, Psychiatry Research and Teaching Unit, Southwest Sydney Local Health District, Sydney, Australia; Wietse Tol, PhD, Dr Ali \& Rose Kawi Assistant Professor, Department of Mental Health, Johns Hopkins Bloomberg School of Public Health, Baltimore, Maryland, USA.

Correspondence: Derrick Silove, Psychiatry Research and Teaching Unit and Ingham Institute, University of New South Wales, Sydney, NSW 2170, Australia. E-mail: d.silove@ unsw.edu.au

First received 19 Jan 2015, final revision 14 May 2015, accepted 27 May 2015

\section{References}

1 Fisher J, de Mello MC, Patel V, Rahman A, Tran T, Holton S, Holmes W. Prevalence and determinants of common perinatal mental disorders in women in low-and lower-middle-income countries: a systematic review. Bull World Health Organ 2012; 90: 139-49.

2 Nguyen $T$, Tran TD, Tran T, La B, Nguyen H, Fisher J. Postpartum change in common mental disorders among rural Vietnamese women: incidence, recovery and risk and protective factors. Br J Psychiatry 2014; 206: 110-5. 
3 Yamamoto N, Abe Y, Arima K, Nishimura T, Akahoshi E, Oishi K, et al. Menta health problems and influencing factors in Japanese women 4 months after delivery. J Physiol Anthropol 2014; 33: 32.

4 Rees S, Silove D, Verdial T, Tam N, Savio E, Fonseca Z, et al. Intermittent explosive disorder amongst women in conflict affected Timor-Leste: associations with human rights trauma, ongoing violence, poverty, and injustice. PLOS One 2013; 8 e69207.

5 Berkowitz L, Harmon-Jones E. Toward an understanding of the determinants of anger. Emotion 2004; 4: 107-30.

6 Silove D, Brooks R, Bateman Steel CR, Steel Z, Hewage K, Rodger J, et al. Explosive anger as a response to human rights violations in post-conflict Timor-Leste. SOC Sci Med 2009; 69: 670-77.

7 Rees S, Silove D, Verdial T, Tam N, Savio E, Fonseca Z, et al. Harmonia Iha Famalia. Women and Anger in Timor Leste. Alola Women's Centre, Timor Leste and the Psychiatry Research and Teaching Unit, UNSW, 2011.

8 Kiernan B. The demography of genocide in Southeast Asia: the deaths tools in Cambodia, 1975-1979, and East Timor, 1975-1980. Crit Asian Stud 2003; 35: 585-97.

9 Dunn J. Genocide in East Timor. In Century of Genocide: Critical Essays and Eyewitness Accounts (eds S Totten, W Parsons): 265-95. Routledge, 2009.

10 Scambary J. Anatomy of a conflict: the 2006-2007 communal violence in Eas Timor. Conflict Security Dev 2009; 9: 265-88.

11 Silove D, Steel Z, McGorry P, Drobny J. Problems Tamil asylum seekers encounter in accessing health and welfare services in Australia. SOC SCi Med 1999; 49 951-56.

12 Silove D, Liddell B, Rees S, Chey T, Nickerson A, Tam N, et al. Effects of recurrent violence on post-traumatic stress disorder and severe distress in conflict-affected Timor-Leste: a 6-year longitudinal study. Lancet Glob Health 2014; 2: e293-300.

13 Liddell B, Silove D, Tay K, Tam N, Nickerson A, Brooks R, et al. Achieving convergence between a community-based measure of explosive anger and a clinical interview for intermittent explosive disorder in Timor-Leste. J Affect Disord 2013; 150: 1242-6.

14 Spitzer RL, Williams JBW, Gibbon M, First MB. The structured clinical interview for DSM-III-R (SCID): I: history, rationale, and description. Arch Gen Psychiatry 1992 49: 624-29.

15 Mollica R, Caspi-Yavin Y, Bollini P, Truong T, Tor S, Lavelle J. The Harvard Trauma Questionnaire. Validating a cross-cultural instrument for measuring torture trauma, and posttraumatic stress disorder in Indochinese refugees. J Nerv Ment Dis 1992: 180: 111-6.

16 Santos JRA. Cronbach's alpha: a tool for assessing the reliability of scales. $J$ Extension 1999; 37: 1-5.
17 Silove D, Manicavasagar V, Mollica R, Thai M, Khiek D, Lavelle J, et al. Screening for depression and PTSD in a Cambodian population unaffected by war: comparing the Hopkins Symptom Checklist and Harvard Trauma Questionnaire with the structured clinical interview. J Nerv Ment Dis 2007: 195: 152-7.

18 Furukawa TA, Kessler RC, Slade $T$, Thai $M$, Khiek $D$, Lavelle J, et al. The performance of the $\mathrm{K} 6$ and $\mathrm{K} 10$ screening scales for psychological distress in the Australian National Survey of Mental Health and Well-Being. Psychol Med 2003; 33: 357-62.

19 Spies G, Stein DJ, Roos A, Faure SC, Mostert J, Seedat S, et al. Validity of the Kessler $10(\mathrm{~K}-10)$ in detecting DSM-IV defined mood and anxiety disorders among pregnant women. Arch Women's Ment Health 2009; 12: 69-74.

20 Bindt $\mathrm{C}$, Appiah-Poku J, Te Bonle M, Schoppen S, Feldt T, Barkmann $\mathrm{C}$, et al. Antepartum depression and anxiety associated with disability in African women: cross-sectional results from the CDS study in Ghana and Côte d'Ivoire. PLOS One 2012; 7: e48396.

21 Schneider M, Baron E, Davies T, Bass J, Lund C. Making assessment locally relevant: measuring functioning for maternal depression in Khayelitsha, Cape Town. SOC PSychiatry PSychiatr Epidemiol 2015; 50: 797-806.

22 Garcia-Moreno C, Jansen HAFM, Ellsberg M, Heise L, Watts C. Prevalence of intimate partner violence: findings from the WHO multi-country study on women's health and domestic violence. Lancet 2006; 368: 1260-69.

23 Kessler RC, Coccaro EF, Fava M, Jaeger S, Jin R, Walters E. The prevalence and correlates of DSM-IV intermittent explosive disorder in the national comorbidity survey replication. Arch Gen Psychiatry 2006; 63: 669-78.

24 Al-Hamzawi A, Al-Diwan JK, Al-Hasnawi SM, Taib NI, Chatterji S, Hwang I, et al. The prevalence and correlates of intermittent explosive disorder in Iraq. Acta Psychiatr Scand 2012; 126: 219-28.

25 Mammen OK, Shear MK, Pilkonis PA, Kolko DJ, Thase ME, Greeno CG. Anger attacks: correlates and significance of an under recognized symptom. J Clin Psychiatry 1999; 60: 633-42.

26 Makieva S, Saunders PT, Norman JE. Androgens in pregnancy: roles in parturition. Human Reprod Update 2014; 20: 542-59.

27 Rees $\mathrm{S}$, Thorpe $\mathrm{R}$, Tol $\mathrm{W}$, Fonseca $\mathrm{M}$, Silove $\mathrm{D}$. Testing a cycle of family violence model in conflict-affected, low-income countries: a qualitative study from TimorLeste. Soc Sci Med 2015; 130: 284-91.

28 vicHealth. National Survey of Community Attitudes towards violence against Women 2009. Changing Cultures, Changing Attitudes - Preventing Violence against Women. Victorian Health Promotion Unit, 2009

29 Hofmann SG, Grossman P, Hinton DE. Loving-kindness and compassion meditation: potential for psychological interventions. Clin Psychol Rev 2011; 31: 1126-32. 